\title{
Microcephaly risk with Zika infection is $1-13 \%$ in first trimester, study shows
}

\author{
Michael McCarthy
}

Seattle

Maternal infection with the Zika virus in the first trimester of pregnancy was associated with an estimated $1 \%$ to $13 \%$ risk of microcephaly, researchers from the US Centers for Disease Control and Prevention have reported. ${ }^{1}$ Their study found "negligible" association between infection and the risk of microcephaly in the second and third trimesters.

The researchers analysed data from Bahia in northeastern Brazil, a region that has been hit hard by the Zika outbreak. They estimated that the risk of microcephaly because of infection in the first trimester ranged from $0.88 \%$ (95\% credible interval $0.80 \%$ to $0.97 \%$ ), assuming an overall Zika virus infection rate among pregnant women of $80 \%$ and $100 \%$ over-reporting of microcephaly cases, to $13.2 \%$ (95\% credible interval $12.0 \%$ to $14.4 \%$ ) if the overall infection rate was only $10 \%$ and there was no over-reporting. The estimate also took account of uncertainty about the baseline rate of microcephaly (between two and 12 cases for each 10000 births).

The researchers, led by Michael Johansson of the CDC's Division of Vector $\square$ Borne Diseases, said, "We found a strong association between the risk of microcephaly and infection risk in the first trimester and a negligible association in the second and third trimesters." However, they found a wide range for estimated risk in the third trimester because the available data were limited in Bahia and other affected areas, where infection rates were unknown and microcephaly cases were still being reported and evaluated.

They cautioned that little was known about the effects of mild or asymptomatic Zika infections, which were probably more common and so may contribute substantially to the overall burden of disease. Microcephaly is also only one possible adverse outcome among a spectrum of conditions that may be part of congenital Zika syndrome, they added.

The researchers concluded, "If the risk of infection and adverse outcomes is similar in the other geographic areas where ZIKV [Zika virus] has since spread, many more cases of microcephaly and other adverse outcomes are likely to occur. In light of the growing evidence, it is prudent to take precautions to avoid ZIKV infection during pregnancy and for health care systems to prepare for an increased burden of adverse pregnancy outcomes in the coming years."

Johansson MA, Mier-Y-Teran-Romero L, Reefhuis J, Gilboa SM, Hills SL. Zika and the risk of microcephaly. N Engl J Med 2016. doi:10.1056/NEJMp1605367. pmid:27222919. Published by the BMJ Publishing Group Limited. For permission to use (where not already granted under a licence) please go to http://group.bmj.com/group/rights-licensing/ permissions 